\title{
The Effects of a Meal on QTc to Demonstrate ECG Assay Sensitivity in Phase I Studies
}

\author{
Jörg Täubel ${ }^{1,2^{\star}}$, Georg Ferber ${ }^{3}$, Sara Fernandes ${ }^{1}$, Ulrike Lorch ${ }^{1}$, Mariano Sust ${ }^{4}$ and A John Camm² \\ ${ }^{1}$ Richmond Pharmacology Ltd., St George's University of London, Cranmer Terrace, London, United Kingdom \\ ${ }^{2}$ Cardiovascular and Cell Sciences Research Institute, St George's University of London, Cranmer Terrace, London, United Kingdom \\ ${ }^{3}$ Statistik Georg Ferber GmbH, Cagliostrostrasse, Riehen, Switzerland \\ ${ }^{4}$ Department of Clinical Investigation, ESTEVE Research \& Development, Av. Mare de Déu de Montserrat, Barcelona, Spain
}

\begin{abstract}
In December 2015, the International Conference of Harmonization (ICH) E14 guideline generated guidance on how exposure-response modelling can be used to characterise the potential for a drug to affect cardiac repolarisation and to modulate the QTC interval. The released Q\&A document describes how data from Phase I ascending dose $S A D$ and MAD studies can be used in the right circumstances to be accepted by regulatory bodies as an alternative approach to TQT to demonstrate cardiac safety. While this strategy is now being expanded to all new drugs, this alternative analysis has been extensively used in the past. This commentary discusses the results of a retrospective analysis where a concentration-effect analysis validated by meal effects on the ECG was applied to a Phase I study to investigate the PK, PD and safety of escalating single doses of a highly selective sigma1 receptor antagonist.
\end{abstract}

Keywords: QTc; Assay sensitivity; Food

\section{Introduction}

The adoption in May 2005 of the ICH E14 guideline established recommendations on the strategy for the clinical evaluation of the propensity of new drugs to cause QTc prolongation [1]. Q\&A documents have been issued with the aim of clarifying any ambiguities concerning the recommendations and/or to consider evidence-based changes and development.

The most recent Q\&A document gives the option of using either Phase I ECG data or data from a TQT study to characterise the potential for a drug to influence QTc as long as high quality ECG recording and analysis are performed [2]. For a study to be considered negative the upper bound of the two-sided 90\% confidence interval for the QTc effect of a drug treatment as estimated by exposure-response analysis should be $<10 \mathrm{~ms}$ at the highest clinically relevant exposure. This revised guidance also states that a separate positive control would not be necessary provided sufficiently high exposure levels are achieved in the early phase study. Realistically, a Phase I study is unlikely to include a pharmacological positive control and in cases where plasma levels of the drug exceeding therapeutic levels are not achieved, the lack of a positive control can constitute a limitation when excluding an effect of regulatory concern. It is therefore important to consider how the study's sensitivity to detect small QT changes can be evaluated if data are to be used as a substitute for a formal dedicated TQT study.

Alternative methodologies to demonstrate assay sensitivity have been considered and discussed by the Cardiac Safety Research Consortium in an attempt to improve the confidence in QT assessment in early-phase studies by reducing the likelihood of false negatives. No solution has been generally accepted to overcome the lack of an approach for assay sensitivity. An acceptable method should be robust and reproducible, easy to implement and must generate small QTc changes and show that these changes can be detected. Other desirable benefits that must not be neglected when choosing an adequate positive control include the possibility of being used in small groups and be applicable across different ethnicities, avoidance of unnecessary drug exposure, absence of anticipated adverse events and no hindrance for ethical approval.

\section{The effect of a meal on QTc}

The effects of food leading to a change in QTc have been successfully demonstrated in different dedicated studies [3-6]. Food if given in a standardised manner, produces a reliable and consistent QTc shortening effect which arises from the postprandial increase cardiac output and the effect of c-peptide and glucose on cardiac repolarisation. Because the effect of a meal can be reliably detected in every subject 2-5 hrs after a meal and on any of the observation days, a separate positive control arm is not a prerequisite nor does the method require subjects treated with placebo. A greater than $90 \%$ power of detecting the meal effects is archived with a sample size of $\geq 8$ subjects if sampling times are dense. No major changes to the study design would be required for the implementation of this test for assay sensitivity as it is based on the same data as the analysis of the drug effect. The study design should only include rigorously standardised and correctly timed meals in addition to sampling of PK and ECG data at appropriate time points.

The requirements for this approach include adequate meal supervision, two to three ECG time points after a meal and subsequent analysis of the pre-meal baseline data compared to the time-points after the first meal of the study day. Therefore, food intake outside the meal times should be controlled and if more than one meal is planned, subjects should be fasting for at least a minimum of $4 \mathrm{hrs}$ but preferably $6 \mathrm{hrs}$ before the meal to avoid overlapped effects in QTc baseline.

If all recommendations above are followed, a standardised meal would be expected to induce a decrease in QTcF of 6-8 $\mathrm{ms}, 2$ to $5 \mathrm{hrs}$

*Corresponding author: $\mathrm{Dr}$ Jörg Täubel, Richmond Pharmacology Ltd, St George's University of London, Cranmer Terrace, SW17 ORE London, United Kingdom, Telephone: +44(0)20 8664 5200, Fax: +44(0)20 8664 5201; E-mail: j.taubel@richmondpharmacology.com

Received October 24, 2016; Accepted October 30, 2016; Published November 10,2016

Citation: Täubel J, Ferber G, Fernandes S, Lorch U, Sust M, et al. (2016) The Effects of a Meal on QTc to Demonstrate ECG Assay Sensitivity in Phase I Studies. J Bioequiv Availab 8: 272-273. doi: 10.4172/jbb.1000307

Copyright: $\odot 2016$ Täubel $\mathrm{J}$, et al. This is an open-access article distributed under the terms of the Creative Commons Attribution License, which permits unrestricted use, distribution, and reproduction in any medium, provided the original author and source are credited. 
Citation: Täubel J, Ferber G, Fernandes S, Lorch U, Sust M, et al. (2016) The Effects of a Meal on QTc to Demonstrate ECG Assay Sensitivity in Phase I Studies. J Bioequiv Availab 8: 272-273. doi: 10.4172/jbb.1000307

after food intake as a result of the relationship between the ratio of glucose and C-peptide concentration effects on QTc [7-9].

\section{Concentration-effect analysis validated by meal effects on QTc}

In a recent publication, the characterisation of the effects of E-52862 on QT interval in healthy subjects was described [4]. The cardiac safety of this selective sigma-1 receptor antagonist was assessed in a double blind randomised placebo-controlled, four-way crossover Phase I study. Subjects received single doses of $500 \mathrm{mg}, 600 \mathrm{mg}$ or $800 \mathrm{mg}$ of E-52862 or placebo. The range of doses tested exceeded the estimated therapeutic dose range of 100-400 mg based on Cmax and the resultant plasma exposures were clearly higher than those expected to be used in efficacy trials [10]. The reported study consisted of a placebo baseline ECG day (Day-1) preceding the respective treatment days. Standardised meals identical in all 4 periods with similar nutritional value were served. However, an important aspect to be highlighted is the fact that at the time of conduction of the trial we were unaware of the effects of a meal on QTc and therefore the study was not specifically designed to use food as positive control. Hence, the use of a concentration effect model to characterise the potential of this drug to influence QTc, utilising effects of a meal to demonstrate assay sensitivity was concluded retrospectively under a supplementary statistical analysis plan which was signed before release of the data to an independent statistician.

The test for assay sensitivity was based on the predicted effect of lunch containing an approximated ratio of $58 \%$ carbohydrate to $22 \%$ fat to $23 \%$ protein; $575 \mathrm{kcal}$ in total. In the analysis of the change from average baseline, two sided $95 \%$ CIs for the differences between the two postprandial time points 1 and $3 \mathrm{~h}$ post-meal, to pre-dose were completely below nought and the point estimate for the difference was well below $5 \mathrm{~ms}$, fulfilling the ICH E14 guideline requirements for the use of a positive control to assess assay sensitivity.

When the effect of food on QTc of this study was compared to the effect seen on a well-controlled TQT study, solely designed to evaluate the effect of a drug effect on cardiac repolarization, both studies showed consistently a food effect present in all periods [6]. Individual QTcF changes $(\triangle \mathrm{QTCF})$ for the 4 periods for both studies also supported the reproducibility of the food effect within each subject. Some subjects could have presented a prolongation effect in one or more periods but the mean food effect on QT was consistently present [6]. This analysis reported by Taubel et al. confirms that the shortening effects on QTc induced by a meal are a reproducible and stable approach to be included in a Phase I setting. This is more challenging as these studies are not dedicated ECG studies and autonomic changes introduce some noise into the measured ECG assessment since subjects are frequently disturbed and mobilised for performance of various studies.

In summary, the analysis reported by Taubel et al. support the use of a concentration-effect as the definite assessment to exclude a regulatory threshold of $10 \mathrm{~ms}$ in Phase I studies validated by the effects of food on QTc. The methodology used reflects the spirit of the most recent E14 recommendations and shows that a positive control can easily be included to gain confidence in the negative results of the drug and provide reassurance against false negatives.

For continued use experimental conditions described must be followed and operating characteristics of the concentration-effect model should be considered [11].

\section{References}

1. ICH Harmonized Tripartite Guideline E14 (2005) The clinical evaluation of QT/ QTc interval prolongation and proarrhythmic potential for non-antiarrhythmic drugs.

2. E14 Implementation Working Group (2015) ICH E14 Guideline: The Clinica Evaluation of QT/QTc Interval Prolongation and Proarrhythmic Potential for Non-Antiarrhythmic Drugs

3. Taubel J, Wong AH, Naseem A, Ferber G, Camm AJ (2012) Shortening of the QT interval after food can be used to demonstrate assay sensitivity in Thorough QT Studies. J Clin Pharmacol 52: 1222-1239.

4. Taubel J, Ferber G, Lorch U, Wang D, Sust M, et al. (2015) Single Doses up to $800 \mathrm{mg}$ of E-52862 Do Not Prolong the QTc Interval - A Retrospective Validation by Pharmacokinetic-Pharmacodynamic Modelling of Electrocardiography Data Utilising the Effects of a Meal on QTc to Demonstrate ECG Assay Sensitivity. PLoS ONE 10(8): e0136369.

5. Taubel J, Ferber G, Izquierdo I (2015) Confirmation of the Cardiac Safety of Rupatadine in a Single Ascending Dose and Multiple Ascending Dose Study in Japanese Healthy Subjects Using Intensive ECG Assessments. Clinical Pharmacology in Drug Development 4(S1) 1-60.

6. Täubel J, Fernandes S, Ferber G (2016) Stability of the Effect of a Standardized Meal on QTc. Ann Noninvasive Electrocardiol.

7. Taubel J, Lorch U, Ferber G, Singh J, Batchvarov V, et al. (2013) Insulin at normal physiological levels does not prolong QTc interval in thorough QT studies performed in healthy volunteers. Br J Clin Pharmacol 75: 392-403.

8. Wahren J, Ekbery K, Jornvall H (2007) C-peptide and neuropathy in type 1 diabetes. Immunol Endor Metab Agents Med Chem7: 69-77.

9. Gordin D, Forsblom C, Ronnback M, Groop PH (2008) Acute hyperglycaemia disturbs cardiac repolarization in type 1 diabetes. Diabet Med 25(1): 101-105.

10. Abadias M, Escriche M, Vaqué A, Sust M, Encina G (2013) Safety, tolerability and pharmacokinetics of single and multiple doses of a novel sigma-1 receptor antagonist in three randomized phase I studies. Br J Clin Pharmacol 75(1):103117

11. Garnett C, Needleman K, Liu J, Brundage R, Wang Y (2016) Operational Characteristics of Linear Concentration-QT Models for Assessing QTc Interva in the Thorough QT and Phase I Clinical Studies. Clin Pharmacol Ther 100(2):170-178. 